\title{
Notes on Meteorological Balloon Mission Planning
}

\author{
András Sóbester* Helen Czerski ${ }^{\dagger} \quad$ Niccolò Zapponi $^{\ddagger}$ Ian Castro ${ }^{\S}$ \\ University of Southampton, Southampton, Hampshire, SO17 1BJ, United Kingdom
}

\begin{abstract}
In the 21st century the high altitude gas balloon remains an indispensable tool in atmospheric science, meteorology and other applications requiring stratospheric observations. A pre-requisite of the effectiveness of many types of balloon operations is an accurate trajectory forecasting capability, complete with appropriate error estimates. This is particularly important in targeted flights, sample return missions or flights of expensive instruments, whose recovery is essential. The ASTRA (Atmospheric Science Through Robotic Aircraft) initiative led to the development of such a forecast model, which is at the centre of the present paper. A key source of error in such models is our incomplete understanding of the drag opposing the rise of balloons in the free atmosphere - here we propose a new, stochastic model based on empirical data derived from thousands of radiosonde flights. We also examine other sources of prediction error affecting the accuracy of the flight path forecast, such as uncertainties in the wind profile and balloon envelope manufacturing variability. A Monte Carlo framework is used to provide probabilistic touchdown point estimates taking these error sources into account. The above elements have been integrated into a web service, which can be used as a flight planning tool - here we review the key features of its architecture.
\end{abstract}

\section{Introduction}

SATELLite soundings and increasingly ubiquitous radar and lidar observations have been the headline addiStions to the atmospheric scientist's list of data sources in recent decades. Alongside such new capabilities, however, instrument packages carried aloft by gas balloons, widely used since the end of the 19th century, are set to remain essential tools both for routine updating of operational numerical weather prediction models and for research observations. The main argument in the favour of such systems is an economic one: their broad altitude range (from the boundary layer to over $40 \mathrm{~km}$ ), over which the accuracy of the instruments is constant, comes at a comparatively low operating cost, especially in the case of light payloads designed for short missions.

In this paper we shall focus on standard, variable volume, rubber weather balloons with highly elastic envelopes (though much of the discussion is applicable to other types of balloons too). While their basic design has changed little in aeronautical terms over the last half century, improved tracking, communications and on-board data processing capabilities, as well as developments in sensor technology have revitalised this old concept and enabled new missions. For example, lightweight and reliable trackers now facilitate the recovery of payloads. In turn, this makes possible:

a) Significant cost savings. In essence, recoverability enables the use of expensive instruments, where the user cannot afford the high attrition rates associated with standard radiosondes. Cost savings could also be made on the latter, if routine recovery was possible ${ }^{a}$ : of the approximately 75000 radiosondes released annually in the US, only about $20 \%$ are found and returned for reconditioning. ${ }^{1}$

b) Sample return missions.

c) Increased resolution. On a recoverable payload the amount of observational data recorded is no longer limited by the (often meagre) bandwidth of the telemetry system.

\footnotetext{
${ }^{*}$ Senior Lecturer, Aeronautics, Astronautics and Computational Engineering Unit, AIAA Member

${ }^{\dagger}$ Research Fellow, Institute of Sound and Vibration Research

${ }^{\ddagger}$ Undergraduate Student, AIAA Student Member

$\S$ Professor, Aeronautics, Astronautics and Computational Engineering Unit

${ }^{a}$ For example through the involvement of the geocaching community, a model already used by some businesses operating unmanned balloon platforms.
} 
d) Reducing the environmental impact of balloon launches. The circuitry, batteries, etc. of some 'fireand-forget' payloads, such as the conventional radiosonde, can become pollutants if left unrecovered.

The effective planning of recoverable payload missions demands an accurate flight simulation capability - for certain research missions the location of the desired landing area can determine the choice of the type of balloon and parachute and the amount of lifting gas used.

Accurate trajectory simulation is usually also the pre-requisite of mobile launch facility based operations, including matching experiments, where consecutive balloon payloads are flown through the same parcel of air at different times (in order to add a time dimension to observations) or when a localised phenomenon (such as an aerosol cloud) has to be observed at a specific location in the upper atmosphere.

Some elements of the flight profile have other uses too - for instance, an accurate model of the rate of ascent in quiescent air can assist in the more accurate derivation of vertical air motion from observed rate of ascent variations. Possible applications include observations of tropospheric updrafts (McHugh et al. ${ }^{2}$ ), estimating gravity wave energies (Wang and Geller ${ }^{3}$ ) or Brunt-Väisälä frequencies (Dolas and Kishore $\left.\operatorname{Kumar}^{4}\right)$.

From the perspective of building an accurate meteorological balloon trajectory prediction model, an understanding of the variation of the drag coefficient of the balloon throughout its ascent is essential. This usually features a drag crisis partway through the ascent, which marks the transition between two boundary layer regimes. In Section III we take an empirical approach to derive a probabilistic picture of the location and magnitude of the drag crisis - we use high resolution flight data to this end, derived from thousands of high altitude soundings ${ }^{\mathrm{b}}$. We also used a large number of soundings to construct the data set that underpins another critical element of the trajectory analysis: the model of the horizontal drift of the balloon as a result of the winds encountered during its ascent. We discuss this in Section IV, along with a number of other engineering and operational factors that influence the flight path. Section V summarizes some of the open questions of the physics of high altitude balloon flight. In Section VI we turn our attention to the architectural details of the web service implementation of the model described here, with Section VII containing a few parting thoughts. Let us begin, however, with a short review of the basic physics of the flight of variable volume meteorological balloons.

\section{Equations of Motion}

It is convenient to separate the motion of a balloon into a horizontal drift component in a plane defined by a latitude $(x)$ and longitude $(y)$ axis and a vertical ascent component (along a $z$ axis). We shall compute the drift based on the Lagrangian assumption that the horizontal motion of the balloon is identical to that of the parcel of air surrounding it, so in an Earth-bound coordinate system

$$
\begin{gathered}
\frac{\mathrm{d} x}{\mathrm{~d} t}=-w(z) \cos [\phi(z)] \\
\frac{\mathrm{d} y}{\mathrm{~d} t}=-w(z) \sin [\phi(z)]
\end{gathered}
$$

where $w(z)$ and $\phi(z)$ are the speed and direction respectively of the wind at altitude $z$ (defined according to the meteorological 'direction from' convention). We shall return to drift modeling in Section IV - for now, let us focus on the ascent component. Its equation is simply a statement of buoyancy opposing weight and drag:

$$
\left(m_{\text {bal }}+m_{\text {pay }}+m_{\text {gas }}\right) \frac{\mathrm{d}^{2} z}{\mathrm{~d} t^{2}}=B-\left(m_{\text {bal }}+m_{\text {pay }}\right) g-D,
$$

where the mass components relate to the balloon, its payload (including strings, parachutes, de-reelers, light sticks, etc.) and the lifting gas. They are assumed to be constant throughout the ascent (that is, no ballast is released). The mass of the lifting gas can be determined by metering during the filling process, though the far more common method is to estimate it indirectly from the nozzle lift $L_{N}$ generated by the

\footnotetext{
${ }^{\mathrm{b} O b t a i n e d ~ f r o m ~ t h e ~ N a t i o n a l ~ O c e a n i c ~ a n d ~ A t m o s p h e r i c ~ A d m i n i s t r a t i o n ~(N O A A) ~ v i a ~ t h e ~ S t r a t o s p h e r i c ~ P r o c e s s e s ~ a n d ~ T h e i r ~}$ Role in Climate (SPARC) project within the World Climate Research Programme (WCRP), URL: http://www.sparc.sunysb.edu.
} 
filled balloon on the ground ${ }^{c}$. If we use the 0 superscript to refer to launch site conditions

$$
m_{\text {gas }}=\rho_{\text {gas }}^{0} \frac{L_{N} / g+m_{\text {bal }}}{\rho_{\text {air }}^{0}-\rho_{\text {gas }}^{0}},
$$

where the densities are calculated using the ideal gas assumption, that is, $\rho_{\text {gas }}^{0}=p^{0} M / R T^{0}$ [with appropriately weighted $M$ values, see equations (7) and (8) in the Appendix]. The buoyancy term of Equation (2) varies with the altitude $z$ (as the volume, pressure, density and temperature all change with altitude) and is computed as:

$$
B=V(z)\left[\rho_{\text {air }}(z)-\rho_{\text {gas }}(z)\right] .
$$

Perhaps the most interesting part of Equation (2) is the drag term. For the purposes of this analysis we define the drag $D$ as the vertical aerodynamic force opposing the buoyancy of the balloon, and the drag coefficient $C_{D}^{b}$ as the dimensionless result of dividing this force by the dynamic pressure of the flow encountered by the balloon and by the projected frontal area $A(z)$ of a sphere with the same volume as the (nearly spherical) balloon. Formally:

$$
D=\frac{1}{2} \rho(z)(\mathrm{d} z / \mathrm{d} t)^{2} C_{D}^{b}(\operatorname{Re}) A(z) .
$$
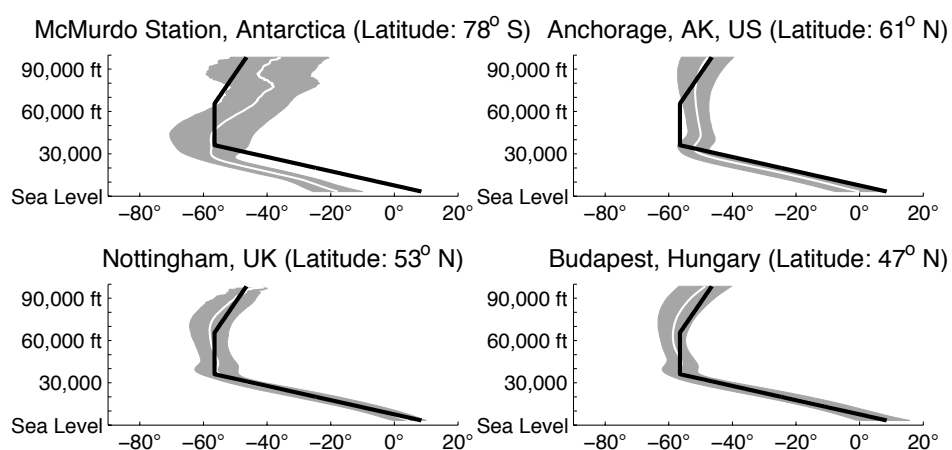

Port Elizabeth, South Africa (Latitude: $34^{\circ} \mathrm{S}$ ) Tucson, AZ, US (Latitude: $32^{\circ} \mathrm{N}$ )
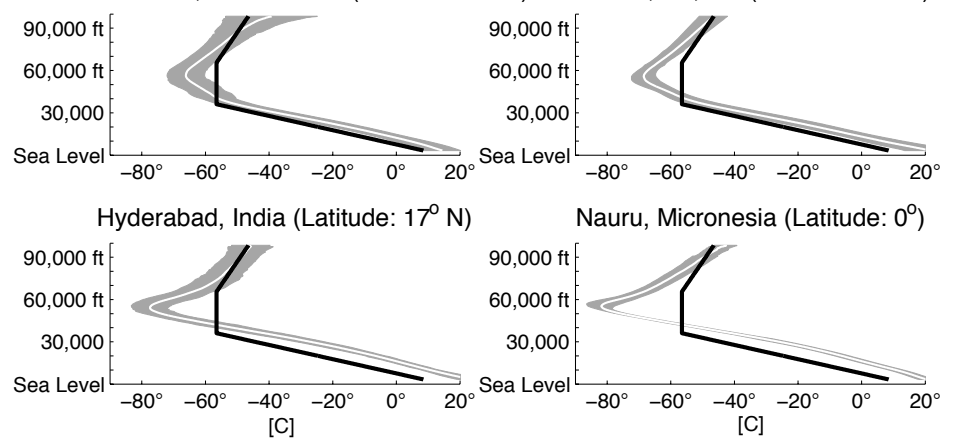

Figure 1. Atmospheric temperatures above eight locations, averaged over the period 1999-2009. The width of the grey band either side of the (white) average temperature curves represents plus and minus one standard deviation respectively. The heavy black lines represent the International Standard Atmosphere model. Reproduced from Ref 5. ulating flights with a variety of gas venting schedules, another major stumbling block in the path of fast analytical solutions.

The vertical motion of the balloon [Equation (2)] thus has to be resolved numerically given the forecast (or actual) atmospheric conditions on the day, as well as taking into account any venting manouvres that

\footnotetext{
${ }^{\mathrm{c}}$ The nozzle lift is the upward force measured on the nozzle of the inflated balloon, without the payload being attached.
} 
may be required. Similarly, the drift equations (1) have to be solved numerically using forecast (or actual) winds.

The time domain integration in both the vertical (ascent) and the horizontal (drift) dimensions is often complicated somewhat by the presence of launch and venting transients, as well as relatively abrupt changes in the environmental conditions (wind- and temperature shears, etc.), which can stiffen the equations. Combined with the need for rapid integration (especially in the light of uncertainties often demanding relatively large Monte Carlo ensembles), this led us to employ LSODA, an ordinary differential equation solver capable of adapting to such changes 'on the fly'. In stiff cases, backward differentiation schemes, such as that implemented in the Livermore Solver for Ordinary Differential Equations (LSODE ${ }^{7}$ ), yield stable solutions, with step size adaptation ensuring high speed. Further speed-ups are possible, however, when a non-stiff case is detected - in such cases LSODA (which is a later development of LSODE) switches to a faster implicit Adams method.

The boundary conditions are provided by the relevant temperature, pressure, humidity and wind profile of the atmosphere [the predictor, to be described in more detail in Section VI, obtains these from NOAA's National Operational Model Archive and Distribution System (NOMADS)], as well as by the ambient conditions and geographical location of the balloon inflation process.

\section{Balloon Drag Estimates}

The wealth of experimental data available on the drag of spherical objects is of limited use when it comes to modelling the drag opposing the ascent of a weather balloon. First, the fluids used in these experiments are somewhat inadequate as surrogates of the free atmosphere. Second, the shape of weather balloons often deviates to some extent from the spherical. Third, these results would need to be corrected for the surface friction drag of increasingly strained latex. Nevertheless, the dependence of the drag of weather balloons on Reynolds number appears to exhibit a feature it shares with smooth spheres: a drag crisis, typically somewhere in the $3 \times 10^{5}$ to $4 \times 10^{5}$ range of Reynolds numbers.

Consider, for instance, the sharp drop in ascent rate evident in Figure 2 (showing the ascent profile of a typical sounding balloon flight) as the balloon passes through this putative transition region. From an average of around $6 \mathrm{~m} / \mathrm{s}$ throughout much of the troposphere, the vertical speed of the balloon begins to fluctuate significantly at an altitude of around $7 \mathrm{~km}$, before settling around $4.5 \mathrm{~m} / \mathrm{s}$ for the next kilometer or so. This period of change in the rate of ascent roughly coincides with the general trend of the Reynolds number (derived here from an estimated balloon diameter variation) intersecting the edges of the $\left[3 \times 10^{5}, 4 \times 10^{5}\right]$ band (shown in grey). This is consistent with a drag crisis occurring in this domain, which the balloon exits with a significantly increased drag coefficient.

While anecdotal evidence of the sort presented in Figure 2 offers little more than an illustration at this point (some other effect may, theoretically, have slowed the ascent in this case), we shall see shortly that this sharp change in the rate of ascent in this particular Reynolds number range occurs consistently across a very large number of flights and therefore adopting a drag model that features a drag coefficient crisis here is, at the very least, practically useful in predicting balloon trajectories.

The physics of the drag crisis could be summarised as follows. The Reynolds number of an ascending weather balloon is dominated by the ambient density and the viscosity, so, in spite of the diameter of the balloon increasing sometimes by an order of magnitude during the ascent, the general Reynolds number trend of the balloon will be a decreasing one. $6 \times 10^{5}$ to $8 \times 10^{5}$ are typical numbers seen early on, in the planetary boundary layer, so the boundary layer of the balloon will be fully turbulent. As the Reynolds number reaches the $3 \times 10^{5}$ to $4 \times 10^{5}$ range (typically about halfway through the troposphere, though the exact height can vary significantly), a laminar region will begin to emerge in the boundary layer near the crown of the balloon. This will have the effect of bringing the boundary layer separation band nearer to the crown and will thus increase drag. 

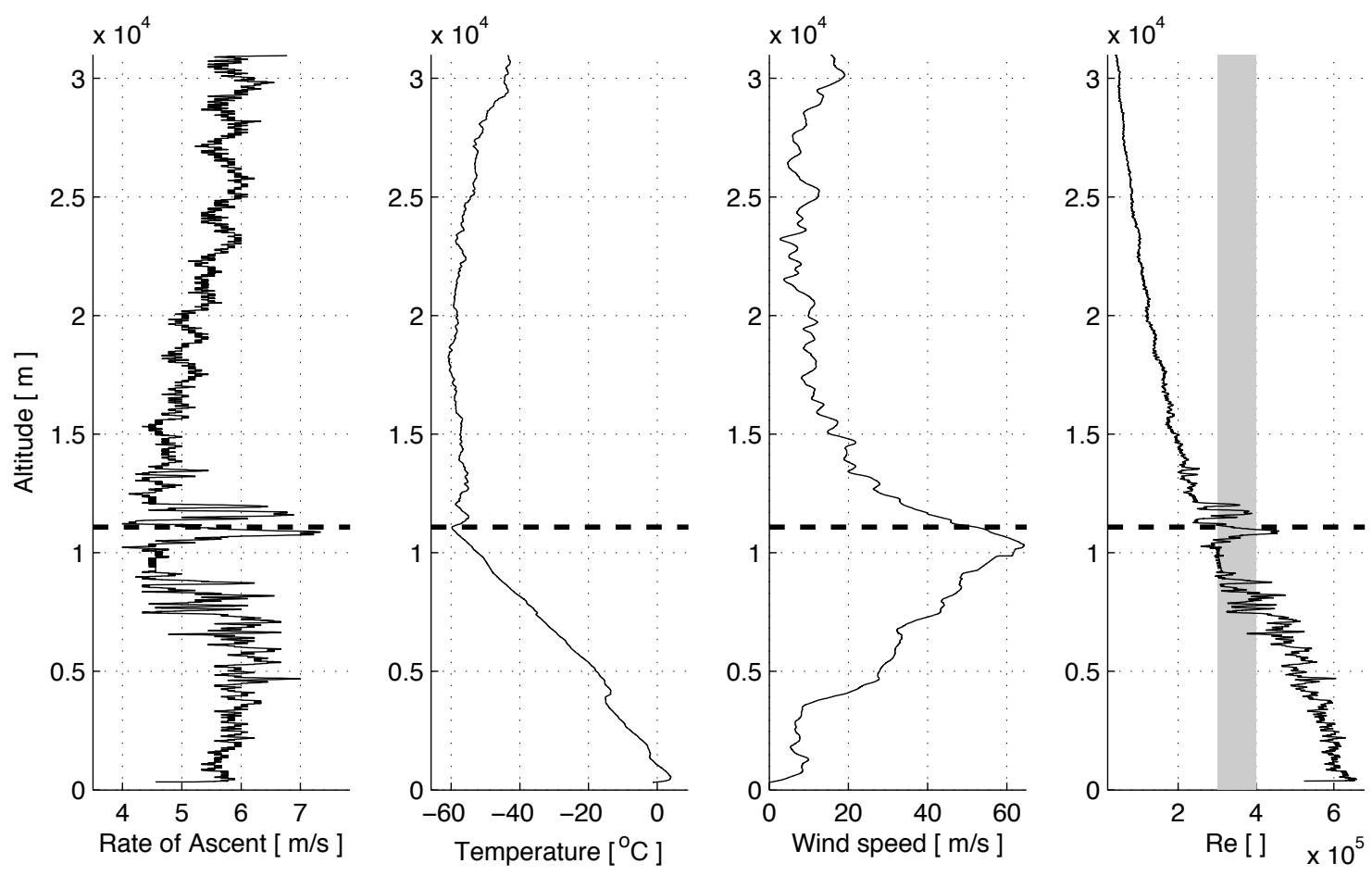

Figure 2. Typical sounding balloon flight. As the balloon passes the transition region (marked by the grey band on the Reynolds number plot), the ascent rate declines significantly. It then increases dramatically for short bursts as the balloon flies through the jetstream, possibly as a result of turbulence accompanying the high gradient shear layers around the jetstream [Wilmington (IL), 15 April 2008, mean tropospheric lapse rate $3.66^{\circ} \mathrm{C} / \mathrm{km}\left(\mathrm{ISA} 6.49^{\circ} \mathrm{C} / \mathrm{km}\right)$, the thick dashed line indicates the location of the tropopause].

In the interest of building a generic balloon flight model, we parameterise the drag variation outlined above and then attempt a probabilistic estimate of these parameters. The complexity and robustness of the parameter estimation process - which we shall pose as an optimization problem - increases (exponentially) with the number of parameters, so we distill the complex physical process of the drag crisis to the simple model shown in Figure 3. More formally, we pos-

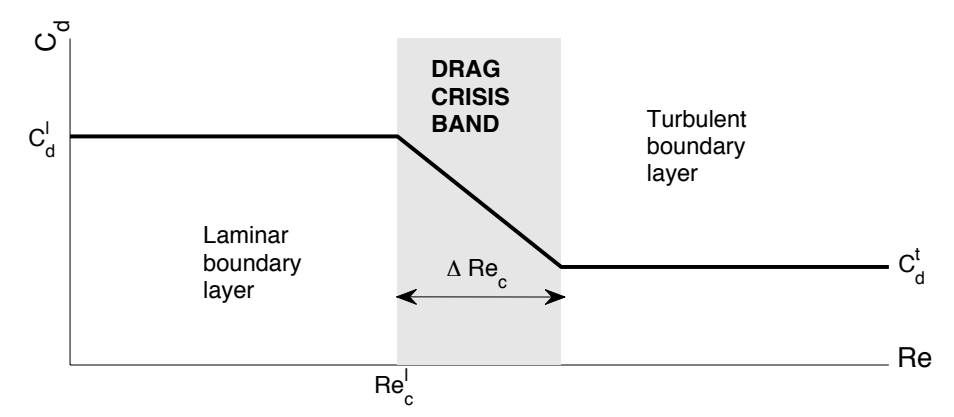

Figure 3. Postulated balloon drag coefficient variation around the drag crisis band. tulate that in the region of interest from the perspective of weather balloon operations, the drag coefficient of the ascending balloon has the form:

$$
C_{d}^{b}\left(\operatorname{Re}, C_{d}^{l}, \operatorname{Re}_{c}^{l}, \Delta \operatorname{Re}_{c}, C_{d}^{t}\right)=\left\{\begin{array}{cc}
C_{d}^{l} & \text { if } \operatorname{Re}<\operatorname{Re}_{c}^{l} \\
C_{d}^{l}-\left(C_{d}^{l}-C_{d}^{t}\right) \frac{\operatorname{Re}^{2} \operatorname{Re}_{c}^{l}}{\Delta \operatorname{Re}_{c}} & \text { if } \operatorname{Re}_{c}^{l} \leq \operatorname{Re} \leq \operatorname{Re}_{c}^{l}+\Delta \operatorname{Re}_{c} \\
C_{d}^{t} & \text { if } \operatorname{Re}>\operatorname{Re}_{c}^{l}+\Delta \operatorname{Re}_{c}
\end{array}\right.
$$

The superscripts $b, l$, and $t$ in the equation above stand for 'balloon', 'laminar' and 'turbulent' respectively, while the subscript $c$ stand for 'crisis'.

In order to estimate the four unknown parameters of Equation (6) we consider a large empirical data set comprising 2093 sounding balloon flights with helium-filled, 700g latex balloons. These were conducted 
by the US National Weather Service at eight different sites ${ }^{\mathrm{d}}$ spread across the United States, representing a range of climatic and topographic conditions. For each flight we compute those values of the four parameters that minimize the (root mean squared) difference between the ascent rate profile predicted by the solution of Equation (2) and the actual ascent rate profile of the flight. As the trajectory simulation for each putative quartet of parameters takes roughly 30 seconds, this is a relatively expensive optimization process - a Nelder-Mead-type simplex heuristic was used.

Figure 4 depicts the histograms of all values of all four parameters recorded during the flight, with the central tile of the image representing the drag model based on the estimated means of each of these distributions (Table 1 records the parameters of these distributions). It is interesting to compare this model with the wind tunnel measurements taken over smooth spheres by Achenbach, ${ }^{8}$ as well as the recent experiments of Son et al. ${ }^{9}$ These data demonstrate the well known fact that the location of the critical transition region is dependent on turbulence levels in the free stream - specifically, the more turbulent the free stream, the lower the critical Reynolds number will be $\mathrm{e}^{\mathrm{e}}$. It is interesting to note that the mean values of the transition location and width resulting from the analysis of the balloon flights are very closely aligned with the $0.45 \%$ turbulence intensity data of Achenbach. ${ }^{8}$ The right hand tails of the distributions of the two transition point values (top and bottom tiles of Figure 4) stretch beyond the transition point observed by Achenbach, indicating atmospheric turbulence levels lower than $0.45 \%$ on a number of flights.

\begin{tabular}{c|cccc} 
& $C_{d}^{l}$ & $\operatorname{Re}_{c}^{l}$ & $\Delta \operatorname{Re}_{c}$ & $C_{d}^{t}$ \\
\hline$\mu$ & 0.225 & $3.296 \times 10^{5}$ & $0.363 \times 10^{5}$ & 0.425 \\
$\sigma$ & 0.036 & $0.511 \times 10^{5}$ & $0.054 \times 10^{5}$ & 0.054
\end{tabular}

Table 1. Results of the model fitting process on 2093 flights with helium-filled, $700 \mathrm{~g}$ balloons.

A note on the interpretation of these results: the distributions are shown merely to provide an intuitive feel for the physical significance of the data (by comparison against available sphere drag measurements) and their depictions are not to be taken to imply that they are statistically independent. For instance, some factor peculiar to a specific launch may equally have affected both the pre-transition and post-transition drag. From the point of view of constructing a Monte-Carlo flight simulator this means that the parameter sets for each point have to be drawn from the joint distribution of the $2093 C_{d}^{l}, \operatorname{Re}_{c}^{l}, \Delta \operatorname{Re}_{c}$ and $C_{d}^{t}$ quartets.

\section{Other Sources of Uncertainty and a Monte Carlo Simulation}

In addition to the uncertainties associated with the ascent drag crisis, a number of other sources of uncertainty widen the inherent error margins of a balloon flight trajectory forecast. The flight planning tool described in this paper accounts for four of these in its error estimates - we discuss them here in turn.

\section{A. Winds Aloft and a Lagrangian Drift Model}

Simulating the drift element of the motion of a weather balloon relies on the assumption postulated in Equation (1) that the horizontal components of the motion are the same as those of the parcel of air surrounding it. Strong vertical wind speed gradients may conspire with the inertia of the payload to violate this assumption, but experience indicates that such effects have a limited impact on the shape of the trajectory.

A more significant cause of trajectory modelling uncertainty lies within the uncertainties associated with the wind vector field itself. Two common sources of wind data used in balloon flight prediction are forecast winds aloft [extracted from a numerical weather prediction model (NWP)] and recent soundings taken in the vicinity of the launch site. While for longer range planning there is no alternative to NWP model data, for launch day analysis the observational data has some advantages, even if they were recorded some distance away from the launch site (both in space and time). Most importantly, soundings extend far into the stratosphere (typically up to about 30-35 km, when taken with a $700 \mathrm{~g}$ balloon) beyond the upper limit of some NWP grids. Their vertical resolution is also higher.

\footnotetext{
${ }^{\mathrm{d}}$ Wilmington (IL), Anchorage (AK), Denver (CO), Newport (NC), Buffalo (NY), Greensboro (NC), Chanhassen (MN) and Quad Cities (IA).

'There is another, more subtle effect: the drag coefficient of a sphere immersed in turbulent flow tends to increase as the integral length scale increases. ${ }^{10}$
} 


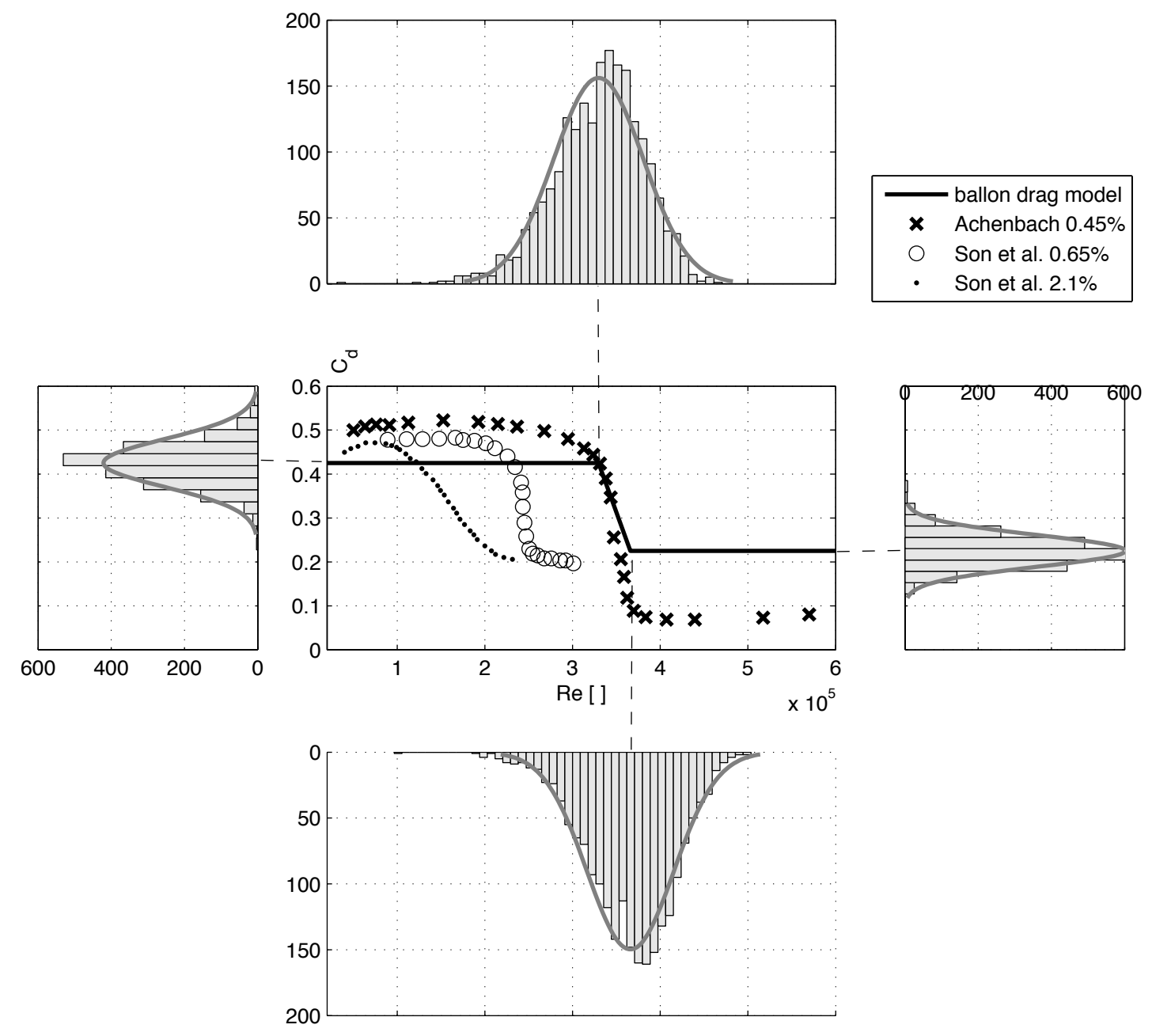

Figure 4. 2093 flights with helium-filled, $700 \mathrm{~g}$ balloons - the continuous line depicts the instance of Equation 6 that results from the sample means of the four distributions 2093 parameter estimates. Also shown are the experimental sphere drag measurements of Achenbach ${ }^{8}$ and Son et al. ${ }^{9}$ taken at various free stream turbulence levels. 
Let us now consider the uncertainties associated with using the observational data of a sounding for the drift calculation element of a balloon flight simulation, arising from the time elapsed since the latest available sounding, as well as from the distance between the launch site of the flight being planned and the location where the sounding was taken. A relatively straightforward way of accounting for these uncertainties in a Monte Carlo framework is as follows:

- We construct a historical database of soundings taken at different sites spaced at mesoscale distances.

- We compute the differences between pairs of neighbouring soundings taken simultaneously, as well as differences between consecutive soundings (typically taken at 12 hour intervals) taken at the same site.

- For each flight in the Monte Carlo simulation we perturb the baseline soundings with a randomly selected entry from this database of variations (scaled with the actual distance between the launch site and the baseline sounding, as well as with the time elapsed between that sounding and the time of launch.

Figure 5 shows the basic statistics of the database (comprising around 3500 historical soundings) we have created for the simulations described in this paper. The two plots show the positive and negative deviations within pairs of soundings, averaged across the whole database. The differences have been computed separately for the $U$ and $V$ components of the wind vectors ${ }^{\mathrm{f}}$ at levels spaced $1000 \mathrm{~m}$ apart up to an altitude of $30 \mathrm{~km}$.

The widening gap between the positive and negative deviations at around $10 \mathrm{~km}$ marks an increasing uncertainty associated with the jetstream (this concurs with the observations of Box et al., ${ }^{11}$ who conducted a similar exercise as part of the development of a rocket flight simulation code).

\section{B. Burst diameter}

In spite of the high quality standards most meteorological balloons are manufactured to, a certain variability and the presence of small flaws (perhaps exacerbated by long pre-flight storage periods) is inevitable. The key metric upon which such geometrical and chemical uncertainties have an impact is the burst diameter of the balloon. Of course, this is just one of the factors that influence the burst diameter (though probably the most important). Others include the inflation technique, pre-launch con-
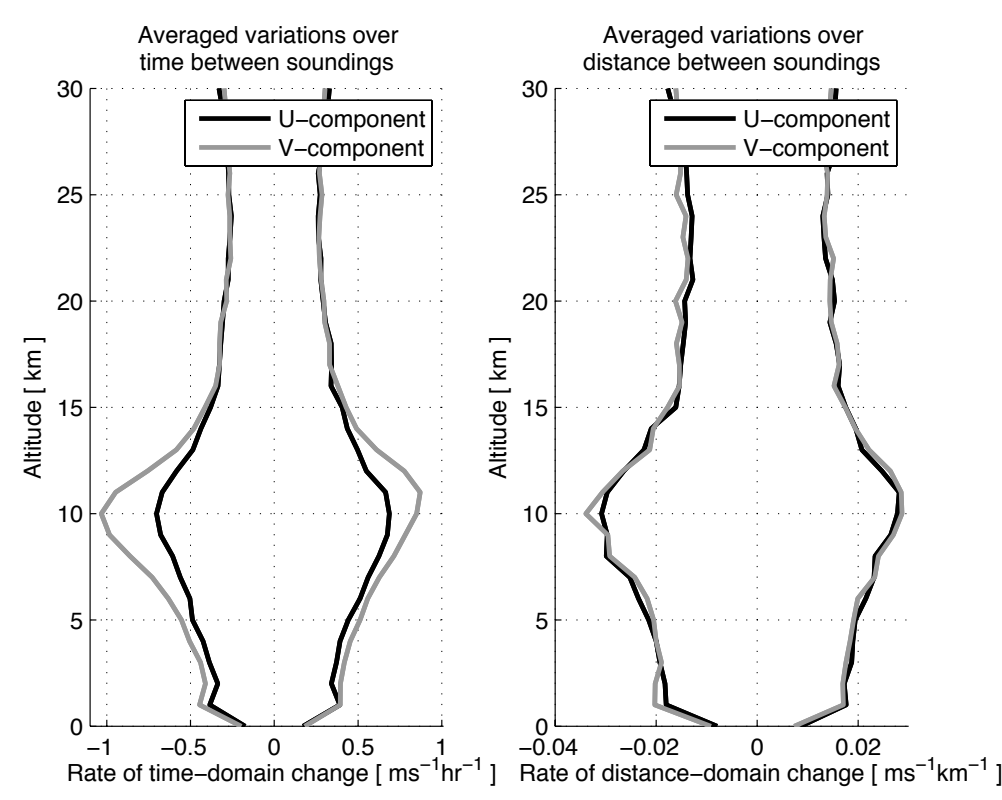

Figure 5. Positive and negative variations between consecutive soundings taken at the same site (left) and simultaneous soundings at neighbouring sites (right), normalised by the time between the soundings and the distance between the launch sites respectively. The data are averaged across a database of around 3500 pairs of soundings taken at various sites. tamination leading to stress concentrations, the payload attachment method, icing, encounters with hail and other forms of precipitation, strong windshears, etc.

In the general uncertainty framework of a balloon flight trajectory forecast model it is thus important to take burst diameter variability into account and, in order to estimate the associated probability density function, we examined 3199 flights conducted with nominally identical 700g balloons filled with Helium and launched by NOAA as part of routine upper atmosphere sounding operations (these flights are part of the same dataset as that used for the drag estimation in Section III).

\footnotetext{
${ }^{\mathrm{f}}$ Using meteorological convention, $U$ represents the East-West component (positive to the East) and $V$ represents the NorthSouth component (positive to the North).
} 
Figure 6 is a histogram of the burst diameters of these balloons as estimated from the key elements of the launch data (inflation temperature and quantity of lifting gas), the environmental conditions recorded by the radiosonde carried by the balloon and the burst altitude. The set has a sample mean of $7.06 \mathrm{~m}$ (comfortably above the manufacturer's nominal value of $6.53 \mathrm{~m}$ ) and a standard deviation of $0.72 \mathrm{~m}$. The Weibull distribution that best fits this data has the parameters $k=14.3577$ and $\lambda=7.3293$, such that the probability density function value (also shown in Figure 6, superimposed on the histogram) for a diameter $d$ is $(k / \lambda)(d / \lambda)^{k-1} e^{\left[-(d / \lambda)^{k}\right]}$.

In order to enable the model to estimate distributions for other bal-

loon sizes too we scaled the distribution found for $700 \mathrm{~g}$ balloons according to the nominal burst diameters of these - this should be a reasonable approximation based on the assumption that the general architecture of the balloon is independent of its size. Figure 7 shows some of these distributions, covering the balloon mass range between $100 \mathrm{~g}$ and $3,000 \mathrm{~g}$.

\section{Descent Uncertainties}

Most high altitude balloon operations involve a parachute descent of the payload. This adds additional uncertainty to flight time estimates, as well as broadening the error margins of the landing zone prediction.

The mechanics of the disintegration of the balloon play an important role here. In most cases the balloon will burst into long, narrow shreds, but the extent to which these shreds separate from the neck of the balloon (to which the payload and the parachute are attached) varies: in some cases all of the rubber remains attached, sometimes only the neck returns. The data available on return fractions is extremely sparse and therefore the best we can do is to assume that the neck will always return (this accounts for about $3 \%$ of the overall mass of the balloon) and the probability of any fraction of the remaining $97 \%$ returning is equal.

For most flights (especially those with very light payloads) this will account for a substantial proportion of the overall descent mass of the train.

Another source of uncertainty on the descent is the drag of the parachute. Based on the available data, as well as on our own experimental observations, we estimate a drag coefficient of between 0.6 and $0.8-$ 
once again, we assume that values within this range are equally probable (the actual value determined by the extent to which the parachute has deployed).

\section{Monte Carlo Ensembles}

The flight path forecasting tool described here is equipped with the ability to generate Monte Carlo ensembles of arbitrary size, based on solving Equations (1) and (2) with the distributions postulated above, as well as on the drag distributions discussed in Section III. The only factor limiting the sizes of these ensembles is the computational budget of the analyst. In our experience 50-100 runs are generally sufficient for practical operational purposes (flight planning), though thousands may be required for rigorous comparative studies.

An example output generated with the code is shown in Figure 8. Ensembles of predicted trajectories were computed five days, three days and one day before a flight, as well as on launch day. The actual trajectory is shown in red on the panels on the left hand side of the Figure 8, with the right hand side depicting landing point density contours. From an operational planning point of view these can be regarded as surrogates for landing probabilities, with the most probable landing zones shown in red.

Touchdown location probability estimation algorithms used in other applications often rely upon the assumption that the distribution of the landing point ensemble is likely to be (close to) Gaussian. Specifically, the contours used in flight planning are those of a normal distribution fitted to the landing point ensemble data. As Figure 8 shows, however, this is not an option in the case of meteorological balloon flight planning, where multimodal landing point distributions are common in Monte Carlo ensembles. Sharp wind shears in the atmosphere are amongst the factors that can split distributions in this way.

\section{Open Questions}

The above discussion hints at the complex interactions that govern the ascent of rubber balloons (contrasting the apparent simplicity of the system!). There is, however, additional complexity in the process, which, for reasons of computational expediency and/or the lack of sufficient experimental evidence, is not accounted for here. Here is a selection of such possible areas needing better uncertainty quantification (largely through a better understanding of the physics involved).

\section{Balloon Geometry}

Most meteorological balloons feature spherical envelopes; that is, they can probably be considered spherical for most practical purposes when neutrally buoyant in still air, unloaded (that is, no payload attached) and inflated at least to the extent that the surface begins to stretch. None of these conditions are present, however, in practical balloon operations in the free atmosphere. While the very reason we employ an empirically derived drag model instead of explicitly assuming sphericity is that we cannot be certain of the exact shape, deviations from a perfect spherical shape have a subtle effect on our analysis when it comes to calculating the frontal area of the ascending balloon (here we do assume a spherical lifting gas bubble). Additionally, a better understanding of the shape variations during the flight may facilitate more germane parameterisations of the drag versus Reynolds number curve, so it is worth considering some of the effects that may play a role in distorting the shape of the balloon.

First, larger balloons (heavier than about 500g) destined for high altitude flights (above $30 \mathrm{~km}$ ) will almost inevitably have to be launched in a flaccid, under-inflated state and they will not reach their fully stretched condition until well into the flight. In these early stages, while the leading half of the balloon will be reasonably well inflated by the bubble of lifting gas within, the trailing half is likely to be more flaccid, potentially exhibiting unsteady behaviour.

Second, the payload train weight vector, applied at the neck of the balloon, may have the tendency to elongate it, as the buoyancy opposing it is more uniformly distributed. However, the frontal pressure associated with the ascent has the opposite (compressive) effect and there is some evidence that the resulting shape is actually an oblate spheroid. ${ }^{4}$

Third, all this is further complicated by dynamic effects induced by possible oscillations. Some of these are likely to be caused by environmental effects, such as zones of vertical or horizontal windshear, internal gravity waves, etc.

The interesting question arising from all this is whether deviations from sphericity have a strong impact on the location and the nature of the drag crisis. In fact, the transition itself, associated with the crown 


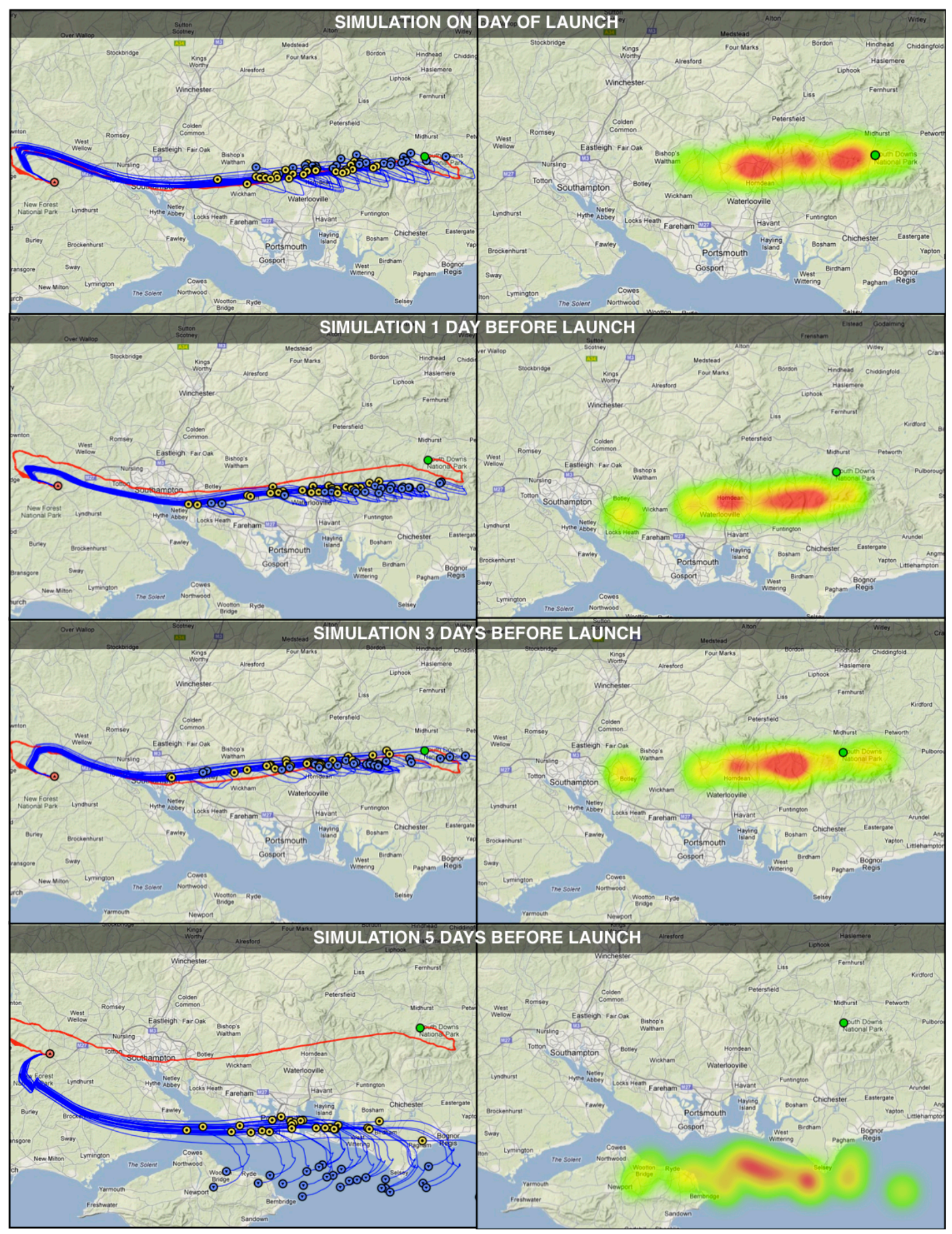

Figure 8. Landing zone predictions computed on launch day (top two panels) and one, three and five days before launch (second, third and bottom rows of panels). The panels in the left hand column show Monte Carlo ensembles of predicted trajectories (in blue), as well as the actual trajectory of the flight (in red). The panels on the right show landing point density contours. The green dot on each panel represents the actual landing site. 
boundary layer being laminar and some of the aft part being turbulent, may, in turn, induce its own distortions.

\section{Thermodynamics}

The analysis presented in this paper neglects a number of thermal effects that may affect the ascent rate of a high altitude balloon. Perhaps the most important of these is the heating of the lifting gas as a result of solar radiation (limited, in practice, by the fact that most meteorological balloons are white).

Additionally, as the latex bubble separating the lifting gas from the ambient air has an extremely thin wall, we assume that the temperature of the lifting gas is uniform and equals that of the ambient air at all times during the ascent. The safety of this assumption clearly depends on the ascent rate, though the critical threshold value (beyond which more sophisticated analysis would be required) is not known. An additional challenge here is the potential computational cost of more detailed thermal analysis, which may have to be traded against the decreased accuracy of smaller Monte Carlo ensembles and therefore a poorer understanding of how uncertainties propagate to the landing site location estimate.

\section{Effusion and Icing}

Another metric of relevance from a balloon dynamics standpoint is the rate of effusion, that is, the rate of passage of the lifting gas through the porosities of the envelope. With the quantity of lifting gas reducing through effusion during the ascent, the buoyancy will drop, and, as a result, so will the rate of ascent. This, in turn, will affect the accuracy of the landing site prediction.

The rate of effusion is inversely proportional to the square root of the molar mass of the gas (Graham's law, see, e.g., Ref. 12). Considering the two lifting gases most frequently used in high altitude balloon applications, the relative effusion rates of helium and hydrogen are thus $\sqrt{M_{\mathrm{He}} / M_{H_{2}}} \approx 1.41$, or, considering the contamination ratios stated in the notes accompanying this paper (see the Appendix), around 1.49. In other words, hydrogen effuses roughly one and a half times faster than helium (with a bond length of $0.74 \AA$, $\mathrm{H}_{2}$ is the smallest molecule $\left.{ }^{13}\right)$.

In either case, actual effusion rates are poorly understood. On the one hand, the impact of the gradual stretching and thinning of the envelope on the effusion rate of either type of gas is unclear, in particular considering the large ambient temperature variations (up to $110^{\circ} \mathrm{C}$ in some cases) in the course of the flight. The effect of other environmental factors also needs further study, both at a microscopic level - in particular, the effect of humidity on the permeability of latex, considering the possibility of very small ice crystals forming in the pores - and at a microscopic level - the possibility of temporary ice sheets accreting across parts of the surface.

Incidentally, both of these mechanisms may also have an effect on the surface roughness and thus on the behaviour of the boundary layer.

Furthermore, it is likely that substantial accretion is limited by the continuous stretching of the latex, a natural de-icing mechanism nearly identical in principle to the de-icing boots employed on medium-altitude propeller-driven aircraft.

\section{The Aerodynamics of the Descent}

We have already hinted at some of the challenges of modelling the descent with uncertain balloon mass fractions. An additional issue here is the uncertain drag of the remains of the balloon, with each remaining strand of latex potentially acting as a streamer during the descent. This is an extremely difficult modelling problem, as both the geometry and the drag coefficient of the burst balloon are highly uncertain.

It is also difficult to account for the possibility of the parachute(s) only deploying partially, as well as for the fact that in some conditions they may be partially immersed in the wake of the payload. With the descent sometimes accounting for as much as $30 \%$ of the overall flight time, this is an important source of uncertainty.

An alternative engineering solution, allowing for more accurate modelling and more mission planning freedom is to keep the balloon intact, by venting much of the lifting gas before the burst altitude is reached, retaining the amount that ensures the buoyancy required for a desired descent rate. 


\section{Web Service Implementation - Computational Architecture}

In order to make the model described here available to the scientific community, a virtual cloud compute server has been set up, capable of running a large number of Monte Carlo ensembles at any given time. With a Windows Azure server processing the simulations, low power devices can be used as user interfaces - including mobile phones and tablets, a useful feature when performing simulations at remote launch sites. Figure 9 shows a screen shot illustrating the graphical interface where the user can specify the launch parameters. Figure 10 is a system diagram illustrating the architecture of the service, which comprises three main components.

The web interface is responsible of interacting with the user, acquiring the input data, requesting a simulation from the server, acquiring the progress of the simulation, loading data, displaying results and grabbing exported data files for the user to download, if required. This is the layer that initiates all actions and informs the server about them.

PHP server scripts are responsible for dealing with requests incoming from the web interface, returning the appropriate results and running server processes. They store the input data, create temporary folders on the server's file system, communicate with the Python back-end to run a flight simulation, inform the web interface of the progress of the simulation and of the file paths to all the output data.

The simulator itself is standalone and works independently of the interface. It re-

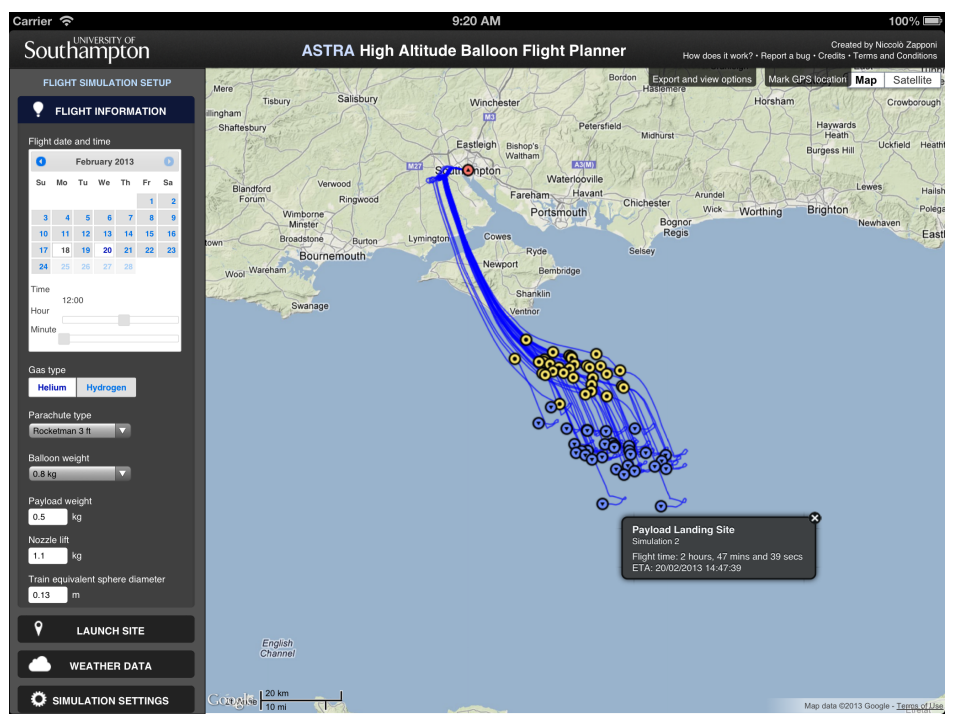

Figure 9. iPad screenshot of the results of a Monte Carlo ensemble simulated using the model described in this paper. ceives input from the PHP scripts, runs the simulation, updates the progress file and stores the results in the server's file system. It can optionally be used with its own Python interface, without using the web, for custom results processing.

\section{In Conclusion}

Much remains to be understood about the complex aerodynamics and thermodynamics of high altitude balloon trains and advances in this direction may, in turn, end up enhancing our understanding of atmospheric processes (mainly through analysing deviations between forecast and actual balloon trajectories). The present paper is a step in this direction, demonstrating how applying some fundamental physical principles and examining large bodies of empirical evidence can help elucidate some of the phenomena involved, while also providing a practical means of forecasting the trajectories of uncontrolled balloon trains.

We make the planning tool described here is free at the point of use - it can be accessed from the ASTRA initiative web pages at www.soton.ac.uk/ ${ }^{\sim}$ astra.

\section{Acknowledgements}

We would like to thank Dr. Steven Johnston and Neil O'Brien for their help with the Azure integration of the flight planner web service and Ben Oxley for providing validation data. Dr. András Sóbester's work was funded by the Royal Academy of Engineering and the Engineering and Physical Sciences Research Council under their Fellowship scheme. 


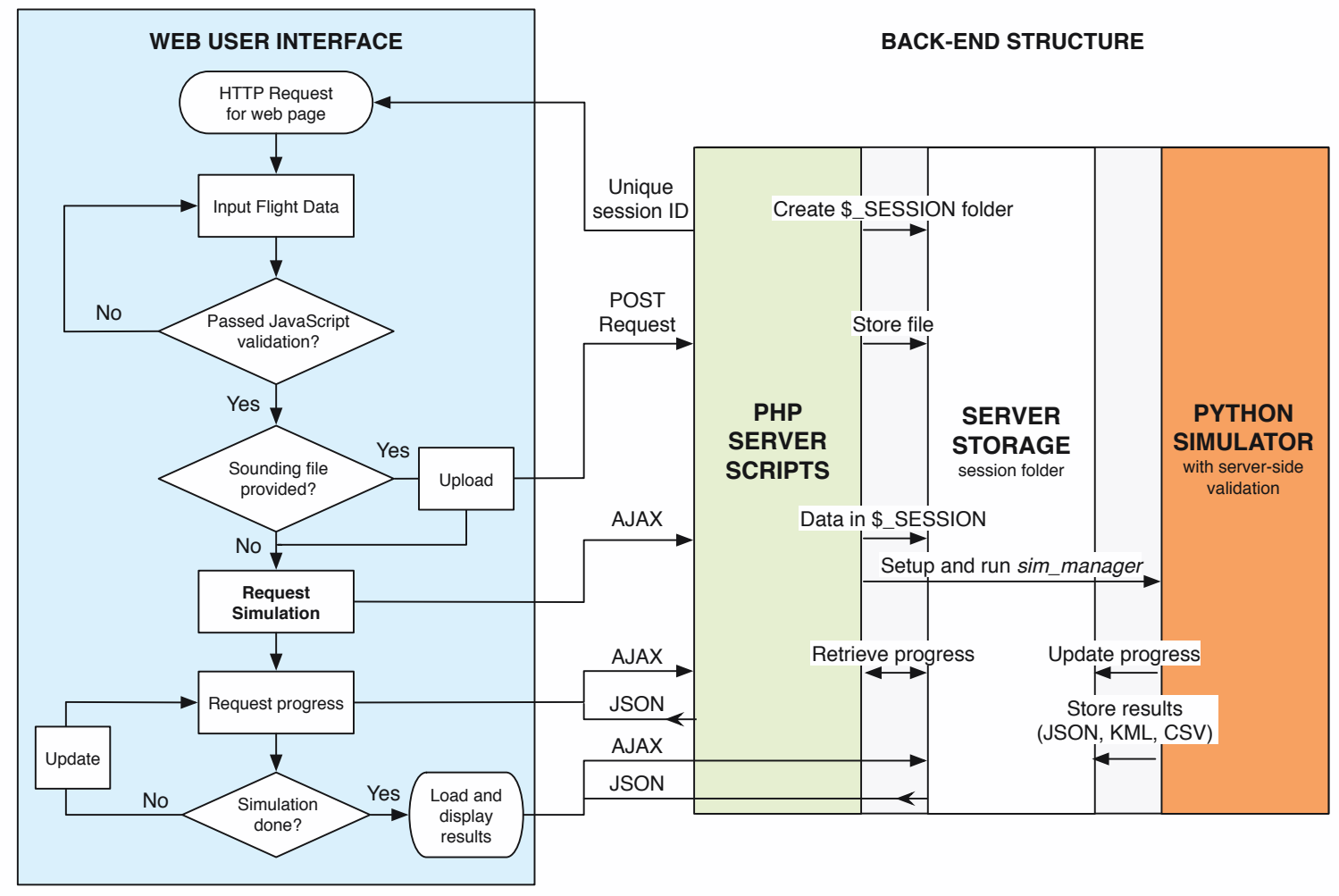

Figure 10. Flight planning tool system architecture. 


\section{Appendix - Notes on Lifting Gases}

The two commonly used meteorological ballooning lifting gases are monatomic helium (He) and diatomic hydrogen $\left(\mathrm{H}_{2}\right)$. They are both an order of magnitude lighter than air. The molecular mass of helium $\left(M_{\mathrm{He}}=0.004 \mathrm{~kg} / \mathrm{mol}\right)$ is roughly twice that of hydrogen $\left(M_{\mathrm{H}_{2}}=0.00201 \mathrm{~kg} / \mathrm{mol}\right)^{\mathrm{g}}$, though this only translates to a minimal performance advantage in hydrogen's favour, as the mass of the lifting gas is usually relatively small compared to the overall mass of a balloon and its payload. Considering balloon grade helium and hydrogen, contaminated with air fractions of $c_{\mathrm{He}}^{\text {air }}=0.055$ and $c_{\mathrm{H}_{2}}^{\text {air }}=0.015$ respectively ${ }^{\mathrm{h}}$ and assuming perfect gas behaviour $\left(R=8.31447 \mathrm{~m}^{3} \mathrm{~Pa} \mathrm{~mol}^{-1} \mathrm{~K}^{-1}\right)$, in ISA sea level conditions $\left(p_{\text {std }}=101325 \mathrm{~Pa}\right.$, $\left.T_{\text {std }}=288.15 \mathrm{~K}\right)$ the densities of the two gases are

$$
\rho_{\mathrm{He}}=p_{\text {std }}\left[\left(1-c_{\mathrm{He}}^{\mathrm{air}}\right) M_{\mathrm{He}}+c_{\text {air }}^{\mathrm{air}} M_{\text {air }}\right] / R T_{\mathrm{std}}=0.22733 \mathrm{~kg} / \mathrm{m}^{3}
$$

and

$$
\rho_{\mathrm{H}_{2}}=p_{\text {std }}\left[\left(1-c_{\mathrm{H}_{2}}^{\text {air }}\right) M_{\mathrm{H}_{2}}+c_{\text {air }}^{\text {air }} M_{\text {air }}\right] / R T_{\mathrm{std}}=0.10235 \mathrm{~kg} / \mathrm{m}^{3} .
$$

Under the same conditions $\rho_{\text {air }}=1.22479 \mathrm{~kg} / \mathrm{m}^{3}$, so for a volume of $V=1 \mathrm{~m}^{3}$ the buoyancy equation yields free lift values of

$$
L_{\mathrm{He}}=V\left(\rho_{\mathrm{air}}-\rho_{\mathrm{He}}\right)=0.99746 \mathrm{~kg}
$$

and

$$
L_{\mathrm{H}_{2}}=V\left(\rho_{\mathrm{air}}-\rho_{\mathrm{H}_{2}}\right)=1.12244 \mathrm{~kg} \text {. }
$$

\section{References}

${ }^{1}$ McGuire, S., "Upper-Air Program Virtual Tour National Weather Service Upper-Air Program, Weather Forecast Office - Reno, NV, url: http://1.usa.gov/rWIXZs," 2011.

${ }^{2}$ McHugh, J. P., Dors, I., Jumper, G. Y., Roadcap, J. R., Murphy, E. A., and Hahn, D. C., "Large variations in balloon ascent rate over Hawaii," Journal of Geophysical Research: Atmospheres, Vol. 113, No. D15, 2008, pp. n/a-n/a.

${ }^{3}$ Wang, L. and Geller, M. A., "Morphology of gravity-wave energy as observed from 4 years (19982001) of high vertical resolution U.S. radiosonde data," Journal of Geophysical Research: Atmospheres, Vol. 108, No. D16, 2003, pp. n/a-n/a.

${ }^{4}$ Dolas, P. M. and Kishore Kumar, K., "Retrieval of static stability parameter from the radiosonde/rawinsonde ascent rate profiles: a wavelet approach," Annales Geophysicae, Vol. 27, No. 2, 2009, pp. 547-553.

${ }^{5}$ Sóbester, A., Stratospheric Flight, Springer, New York, 2011.

${ }^{6}$ Abe, T., Imamura, T., Izutsu, N., and Yajima, N., Scientific Ballooning Technology and Applications of Exploration Balloons Floating in the Stratosphere and the Atmosphere of Other Planets, Springer, 2004.

${ }^{7}$ Radhakrishnan, K. and Hindmarsh, A. C., "Description and Use of LSODE, the Livermore Solver for Ordinary Differential Equations," Tech. Rep. Reference Publication 1327, NASA, Lawrence Livermore National Laboratory,Livermore, CA, 1993.

${ }^{8}$ Achenbach, E., "Experiments on the flow past spheres at very high Reynolds numbers," Journal of Fluid Mechanics, Vol. 54, No. 03, 1972, pp. 565-575.

${ }^{9}$ Son, K., Choi, J., Jeon, W.-P., and Choi, H., "Effect of free-stream turbulence on the flow over a sphere," Physics of Fluids, Vol. 22, No. 4, APR 2010.

${ }^{10}$ Moradian, N., Ting, D., and Cheng, S., "The effects of freestream turbulence on the drag coefficient of a sphere," Experimental Thermal and Fluid Science, Vol. 33, 2009, pp. 460-471.

${ }^{11}$ Box, S., Bishop, C. M., and Hunt, H., "Stochastic Six-Degree-of-Freedom Flight Simulator for Passively Controlled High-Power Rockets," Journal of Aerospace Engineering, Vol. 24, No. 1, 2011, pp. 31-45.

${ }^{12}$ Reger, D. L., Chemistry Principles and Practice, Brooks/Cole Cengage Learning, 2010.

${ }^{13}$ DeKock, R. L. and Gray, H. B., Chemical structure and bonding, University Science Books, 1989.

\footnotetext{
$\mathrm{g}_{\text {air }}=0.02896 \mathrm{~kg} / \mathrm{mol}$.

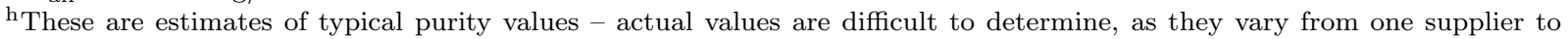
another. They are also influenced by the balloon inflation technique, the amount of residual air contained in the filling device and the balloon itself, etc. and there may therefore also be some variation from one launch site to another.
} 